\title{
Synthesis of highly stable silver nanoparticles by photoreduction and their size fractionation by phase transfer method
}

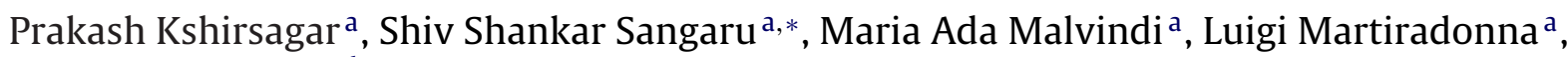 \\ Roberto Cingolani ${ }^{a, b}$, Pier Paolo Pompa ${ }^{a, * *}$ \\ a Istituto Italiano di Tecnologia, Center for Bio-Molecular Nanotechnology @UniLe, Via Barsanti, 73010 Arnesano, LE, Italy \\ ${ }^{\mathrm{b}}$ Istituto Italiano di Tecnologia, Central Research Laboratories, Via Morego, 30-16136 Genova, Italy
}

\section{A R T I C L E I N F O}

\section{Article history:}

Received 9 August 2011

Received in revised form

30 September 2011

Accepted 4 October 2011

Available online 12 October 2011

\section{Keywords:}

Silver

Photoreduction

Tyrosine

Nanoparticles

Laser

UV irradiation

\begin{abstract}
A B S T R A C T
In this report we demonstrate a green chemical approach for the synthesis of stable silver nanoparticles in aqueous medium using tyrosine as an efficient photoreducing agent. A narrow size distribution of silver nanoparticles can be achieved by this simple photoirradiation method without using any additional stabilizing agents or surfactants. Two different irradiation sources have been explored resulting in a different particle size distribution pattern in each case. Further, we show that starting from a polydisperse tyrosine synthesized silver nanoparticles sample, it is also possible to fractionate them into different size ranges. The size fractionation was achieved by a 2 stage phase transfer method employing different organic solvents. The nanoparticles synthesized were characterized using UV-vis spectroscopy, Transmission electron microscopy (TEM) and X-ray diffraction (XRD) techniques.
\end{abstract}

(c) 2011 Elsevier B.V. All rights reserved.

\section{Introduction}

In the recent phase of rapid developments in nanotechnology, metal nanoparticles (NPs) have created a niche for themselves in this field. Metal NPs have interesting plasmonic [1], chemical [2-4], and physical $[4,5]$ properties that have been suitably exploited for various optical [6], electronic [7,8], catalytic $[2,9]$ and biological [10] applications. The current level of understanding of the size and shape dependent nanoscale properties and their wide applications are beginning to impose tougher demands on the availability of efficient synthesis protocols of NPs. Most of the studies and applications now require that the NPs are of narrow distribution with respect to their size and shape. Similarly, NPs synthesized in organic medium or in emulsions might not always suite certain biological applications where the presence of surfactants or strongly binding non-polar organic capping agents could be undesirable. It is thus necessary to have multiple options for synthesizing NPs to fulfill the various requirements demanded by specific applications. Although, good quality gold NPs with controlled

\footnotetext{
* Corresponding author. Tel.: +39 0832 295734; fax: +39 0832295708

** Corresponding author. Tel.: +39 0832295714.

E-mail addresses: shiv.sangaru@iit.it (S.S. Sangaru), pierpaolo.pompa@iit.it (P.P. Pompa).
}

size, shape and functionality can be routinely synthesized using several reported protocols, the number of successful attempts with silver nanoparticles (AgNPs) synthesis is not so impressive. In spite of their growing diverse applications, such as, for Surface enhanced Raman spectroscopy [11], biodetection [12], cell imaging [13], 2D membrane profiling [14], antimicrobial activity [15], catalysis [16], recording media [17], etc., there are comparatively very less reports for facile and efficient synthesis of AgNPs with narrow size distribution. Organic medium based methods [18-20] have been relatively more successful in achieving good monodispersity. In few other cases the initially formed polydisperse NPs are treated post-synthesis with specific strong surface active molecules at elevated temperatures to narrow down the polydispersity by digestive ripening [21,22]. Synthesis has also been made in biphasic systems [23], in polyols [24], and even in ionic liquids [25]. AgNPs with low dispersion have also been synthesized in selected aqueous solutions based methods. To achieve homogeneous nucleation and growth, the synthesis has been preferably done by microwave [26], photoreduction [27,28], laser ablation [29] or in a controlled flow reactor [30]. Alternatively, reduction of saturated solution of $\mathrm{AgCl}$ [31] or reduction under pressurized hydrogen atmosphere [32] has also been carried out to promote growth of monodisperse NPs. However, in most cases, polymeric molecules or other macromolecules have been invariably used additionally to stabilize them. 
In this work an efficient synthesis of AgNPs by a simple photoreduction method using tyrosine as the photoreducing and stabilizing agent has been demonstrated. Tyrosine has been previously used to synthesize AgNPs by a thermal reduction method [33]. However, the AgNPs synthesized thermally had a broad bimodal particle size distribution and hence cannot be put to use where monodisperse samples are necessary. Similarly, oligopeptides of tyrosine too have been used for the synthesis of silver nanoparticles which again apparently yield nanoparticles over a broad range from less than $10 \mathrm{~nm}$ to around $50 \mathrm{~nm}$ [34]. We have attempted to carry out the photoreduction by using two different sources that yield in a narrow size distribution. In the first method, photoreduction was carried out by using the fourth harmonic $(266 \mathrm{~nm})$ of a $\mathrm{Nd}$ :YAG laser. In the second method, a simple and low-power $(4 \mathrm{~W})$ handheld longwave ultraviolet lamp (UV-A lamp) was used for the photoreduction. The NPs synthesized were very stable without any signs of aggregation. The use of the amino acid tyrosine, as a photoreducing agent in absence of any additional stabilizing agent, is a good example of green chemical approach for the AgNPs synthesis. The absence of macromolecules or surfactants renders them suitable for easy surface modifications and for a variety of applications. Furthermore, using a polydisperse sample of AgNPs, we have also demonstrated the possibility of fractionating them into two size ranges by employing phase transfer method.

\section{Experimental}

\subsection{Materials}

Silver sulphate (99\%), L-tyrosine (99\%) and potassium hydroxide (99.99\%) were all purchased from Sigma-Aldrich chemicals. All reagents were used without any further purification. Deionized water was used for the preparation of all aqueous solutions.

\subsection{Synthesis by photoirradiation}

In a typical experiment, a $20 \mathrm{~mL}$ aqueous solution with deionized water consisting of tyrosine $\left(2 \times 10^{-4} \mathrm{M}\right)$ and silver sulphate $\left(1 \times 10^{-4} \mathrm{M}\right)$ was freshly prepared. To this solution $200 \mu \mathrm{L}$ of $0.1 \mathrm{M}$ aqueous $\mathrm{KOH}$ solution was added under stirring condition for $5 \mathrm{~min}$. The same solution was then used for further photoirradiation experiment using either the laser or handheld UV-A lamp.

Laser irradiation was performed using the fourth harmonic $(\lambda=266 \mathrm{~nm})$ of a Spectrophysics Nd:YAG (PRO-250) laser. The pulse rate of the laser was $10 \mathrm{~Hz}$ with a pulse width of $7 \mathrm{~ns}$, and the laser power was set at $80 \mathrm{~mJ} /$ pulse. The laser beam was aligned in order to irradiate a sample solution in a quartz cuvette vertically from the top opening. The irradiation was carried out for $45 \mathrm{~min}$.

The second type of irradiation was carried out using a spectroline handheld lamp consisting of a $4 \mathrm{~W}$ UV-A lamp by placing it $5 \mathrm{~cm}$ above an uncovered $25 \mathrm{~mL}$ glass beaker filled with $10 \mathrm{~mL}$ of the above prepared solution. The irradiation was carried out overnight. For monitoring the kinetics of the reaction with UV-vis spectroscopy, around $1 \mathrm{~mL}$ of the reaction solution was taken at regular intervals for the UV-vis measurements and poured back into the reaction solution within $1 \mathrm{~min}$. As the reaction progressed slowly, this mode of measurement did not influence the reaction process (the end product was similar with identical optical characteristics as observed with an undisturbed photoirradiation reaction). The intensity of the lamp, was measured to be $0.97 \mathrm{~mW} / \mathrm{cm}^{2}$ at a distance of $5 \mathrm{~cm}$. With a back of the envelop calculation, the intensity of laser over a minute of irradiation (considering its pulse rate, $10 \mathrm{~Hz}$ ) would be around 820 times higher than the UV-A lamp. However, the instantaneous intensity of laser during each $7 \mathrm{~ns}$ pulse would be around $6.8 \times 10^{9}$ times that of the UV-A lamp.
As a control experiment for comparison, AgNPs were also synthesized following the already reported thermal method [33]. Briefly, a $50 \mathrm{~mL}$ solution with final concentration of $2 \times 10^{-4} \mathrm{M}$ tyrosine and $1 \times 10^{-4} \mathrm{M}$ silver sulphate in distilled water was prepared. While stirring vigorously, $500 \mu \mathrm{L}$ of $0.1 \mathrm{M} \mathrm{KOH}$ was added at once. This mixture was heated under reflux conditions until the colorless solution changed into yellow color typical of AgNPs and then allowed to cool to room temperature.

For phase transfer experiments, polydisperse AgNPs synthesis was carried out in a UV Laboratory reactor system 2 (UV Consulting Peschl) with a $150 \mathrm{~W}$ medium pressure mercury vapour lamp (Heraeus TQ-150). Around $650 \mathrm{~mL}$ of reaction solution with similar molar ratios and concentrations as in previous cases was used for the synthesis with this lamp.

\subsection{Characterization}

The optical absorbance spectra of the different solutions were measured on a Cary 300 UV-vis spectrophotometer at a resolution of $1 \mathrm{~nm}$ using a $5 \mathrm{~mm}$ path length quartz cuvettes. The NPs were imaged using Transmission Electron Microscopy (JEOL Jem1011, operating at an accelerating voltage of $100 \mathrm{kV}$ ). The samples for analysis were prepared by drop casting the NPs solution on a carbon-coated copper grids (Formvar/Carbon $300 \mathrm{Mesh} \mathrm{Cu}$ ) and drying them under vacuum. Particle size distribution was calculated by measuring at least 200 NPs for AgNPs synthesized by each method, and the resultant histograms were fitted by Gaussian function to obtain the mean value and its standard deviation unless otherwise stated. X-ray diffraction (XRD) measurements were carried out on a Panalytical X'Pert PRO diffractometer with a $\mathrm{Cu} \mathrm{K} \alpha$ (1.54 $\AA$ ) radiation in grazing incidence mode. The samples were prepared by drop casting the aqueous AgNPs solution on a miscut silicon wafer and drying in air. For determining the size of the NPs samples, analysis of the XRD data was done using Origin software. (111) Bragg reflection peaks were fitted using the in-built Lorenztian equation to determine the peak width for calculating the size using the Scherrer equation (1) [35]:

$D_{h k l}=\frac{K \lambda}{\beta_{h k l} \cos \theta}$

where $D_{h k l}$ is the apparent crystallite size along the $[h k l]$ direction, $K$ is the shape factor $(=0.94$ in this case), $\lambda$ is the $X$-ray wavelength, $\beta_{h k l}$ is the half width at half maximum for the $(h k l)$ diffraction peak in radians, and $\theta$ is the half-scattering angle corresponding to the $(h k l)$ diffraction peak.

\section{Results and discussion}

The first type of synthesis of AgNPs by photoreduction in an alkaline tyrosine solution was mediated by the 4th harmonic $(\lambda=266 \mathrm{~nm})$ of a Nd:YAG laser. The reduction and synthesis appeared to occur within the first $30 \mathrm{~s}$ of irradiation as judged visually from the appearance of faint yellow color characteristic of AgNPs. However, the colour initially developed locally within the upper half of the reaction solution in the cuvette, i.e., where the laser beam first encountered the reaction solution, and then proceeded to the lower parts of the cuvette. The solution was irradiated for $45 \mathrm{~min}$ to be sure that the reduction was complete within the entire solution. By irradiating the solutions for more than $50-55 \mathrm{~min}$ with a power of $80 \mathrm{~mJ} /$ pulse, it was observed that the NPs tend to become unstable in the solution. We attribute this loss of stability of the NPs to the complete photodegradation of tyrosine molecules and of their initial reaction products necessary for stabilizing the NPs. Dashed curve and curve (a) of Fig. $1 \mathrm{~A}$ are the UV-vis spectrum of the AgNPs solution measured after $30 \mathrm{~s}$ and 45 min of irradiation, respectively. After 30 s of irradiation a broad 

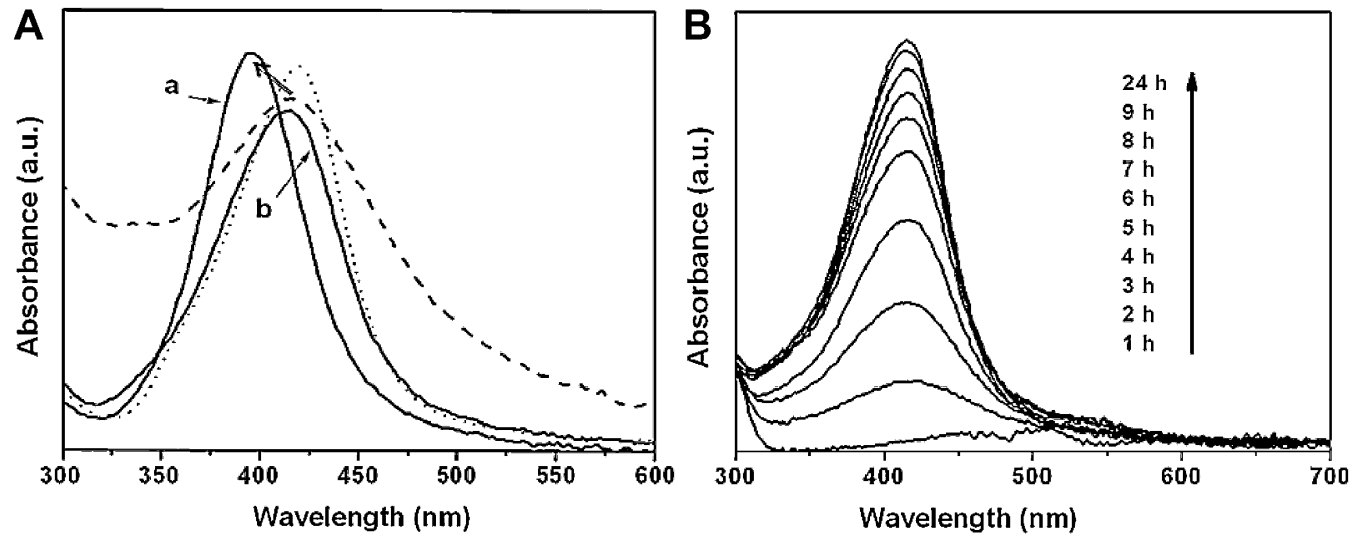

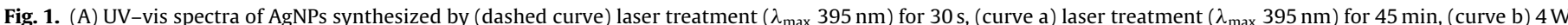

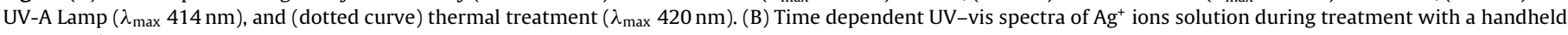
4W UV-A lamp.

absorbance peak is observed at $416 \mathrm{~nm}$ which shifts to $\sim 395 \mathrm{~nm}$ and absorbance band is also observed to be more narrow. From various trials in different conditions, the appearance of an absorbance peak below $400 \mathrm{~nm}$ for aqueous solution of AgNPs corresponded to the absence of any bigger size NPs or aggregates.

In a similar way, reduction of silver ions for the synthesis of AgNPs was also affected by using a handheld $4 \mathrm{~W}$ UV-A lamp under a dark enclosure. Curve (b) of Fig. 1A shows the spectrum of the AgNPs solution formed after overnight irradiation. The absorbance peak was observed to be at $\sim 414 \mathrm{~nm}$ indicating the formation of NPs larger than the ones formed by laser irradiation. For comparison, UV-vis spectrum of AgNPs synthesized by the thermal reduction method with absorbance peak at $420 \mathrm{~nm}$ is also shown (dotted curve, Fig. 1A). Owing to the low power UV-A lamp used for the photoreduction, the formation of AgNPs was much slower, and the reaction was almost complete by $9 \mathrm{~h}$ at room temperature. The evolution of the optical spectra over time can be seen in Fig. 1B; it can be noticed that the rapid phase of particle formation is within $1-5 \mathrm{~h}$ of the reaction, with slow incremental differences occurring there after. From the UV-vis kinetics (Fig. 1B) it can be observed that the intensity of the absorbance peak constantly increases without any noticeable change in its peak position. This suggests that the reaction does not occur via the formation of small seed NPs in the beginning that further grow into larger particles. In a control experiment carried out in parallel, formation of AgNPs could not be observed in a similar reaction mixture left at room temperature in the dark for the same time period. However, some reduction was observed to occur when left in ambient light resulting in a very polydisperse AgNPs (Fig. S1A).

In Fig. 2A TEM analysis of the AgNPs synthesized by laser photoreduction is reported. Clearly, the AgNPs are mostly of size less than $10 \mathrm{~nm}$, they are well dispersed and seem to have a narrow size distribution. In panel (a) of Fig. 2C, the particle size distribution calculated from the TEM images is shown. From the fitted curve of the histogram, the mean size of the AgNPs synthesized by laser irradiation is found to be $\sim 8 \mathrm{~nm} \pm 2 \mathrm{~nm}$. Similarly, in Fig. 2B, the AgNPs synthesized by UV-A lamp photoreduction is shown. As it could be also deduced from the UV-vis spectra (curve b, Fig. 1A), these NPs were of larger size as compared to laser irradiation. After fitting the TEM particle size distribution, the mean particle size was calculated to be $19 \pm 3.8 \mathrm{~nm}$ (panel b Fig. 2C). The larger size of these AgNPs can be attributed to the low intensity of the UV-A lamp that results in a slow reduction and hence slow rate of nucleation. Time dependent TEM (Fig. S2) revealed that initially there are large percentage of AgNPs of size around $10 \mathrm{~nm}$ up to $3 \mathrm{~h}$ of irradiation. There percentage gradually decreases with time, though they continue to exist noticeably even up to $5 \mathrm{~h}$ of irradiation (during the time that rapid changes in absorbance was observed). It therefore can be seen that in these irradiation conditions there are no separate nucleation and growth phases for AgNPs formation. However, beyond $5 \mathrm{~h}$ of irradiation, the nucleation rate decreases considerably and apparently preferential growth of smaller AgNPs occurs leading to a size distribution between 12 and $25 \mathrm{~nm}$. In the case of laser irradiation, due to the high intensity of the beam $(80 \mathrm{~mJ} /$ pulse $)$, the reduction occurs rapidly and the changes can be seen visually in short time. This rapid reduction gives less chance for the growth of initially formed NPs and favors the formation of smaller NPs due to enhanced rate of nucleation. Moreover, the laser beam also tends to fragment the NPs that grow into bigger size [36]. It is observed that though after $30 \mathrm{~s}$ of irradiation few particles of size $20-30 \mathrm{~nm}$ are formed initially (Fig. S3), they are not present after $45 \mathrm{~min}$ of irradiation. Hence, under these conditions, a size focusing could also be occurring to yield NPs with narrow size distribution. In fact, from the histograms in Fig. $2 \mathrm{C}$, it can be noticed that the AgNPs synthesized by both the photoreduction methods have much narrower size distribution as opposed to the broad size distribution obtained by thermal reduction method [33]. Under thermal reduction conditions, especially during the temperature ramp up stage, nucleation is very likely initiated in the solution prior to the attainment of boiling temperature. This results in rapid growth of the nuclei formed at ramp up stage compared to those formed in boiling conditions, leading to the observed polydisperse sample. Tyrosine and similar molecules with phenol moieties are well known to release electrons with simultaneous formation of phenoxyl radicals in aqueous solutions upon UV irradiation $[37,38]$. The reduction of silver ions, $\operatorname{Ag}(\mathrm{I})$ to $\mathrm{Ag}(0)$ could occur through this radical formation mechanism. As under photoirradiation conditions there is a better chance for homogeneous photoactivation, uniform reduction in solution can be achieved very conveniently. Further, the phenoxyl radicals are formed more efficiently via photoionization of deprotonated phenoxide ion under alkaline conditions than at lower $\mathrm{pH}$ [34,39], in fact, also in our attempts AgNPs synthesis was not successful at neutral pH. From FTIR characterization (Fig. S4) a peak at around $1630 \mathrm{~cm}^{-1}$ is observed that can be attributed to a carboxylate anionic group rather than to a ketonic group that occurrs at a relatively higher frequency. Most likely the reduction proceeds via formation of dityrosine like radical adducts [34] rather than semiqunione derivatives as occurring in thermal reduction [33]. Additionally, it may be noted here that as the nitrate ions are good scavengers of free electrons they significantly inhibit the photoreduction reduction process, hence, we used $\mathrm{AgSO}_{4}$ as a precursor instead of the more soluble $\mathrm{AgNO}_{3}$ salt. However, on the contrary, 

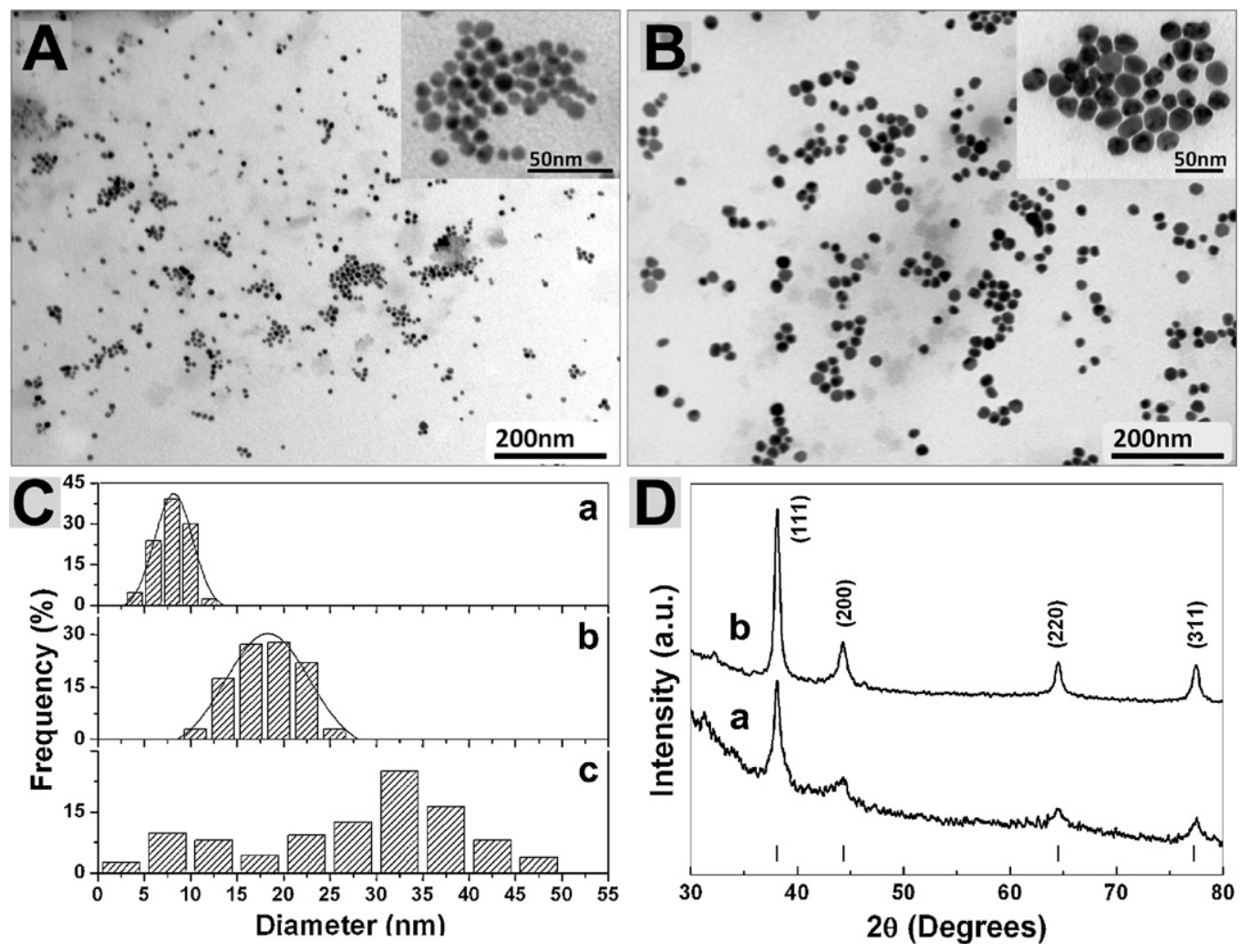

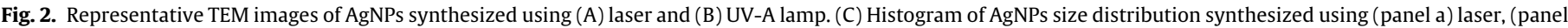

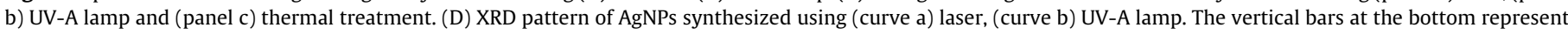
the $2 \theta$ values for standard fcc silver (JCPDS 04-0783).

when the reaction was carried out in nitrogen atmosphere with degassed precursor solutions, the AgNPs were more polydisperse with a significant presence of NPs size $\leq 10 \mathrm{~nm}$ (Fig. S1B). The AgNPs synthesized by photoirradiation were also characterized by X-ray diffraction (XRD). The NPs samples were all observed to be crystalline with their Bragg reflection patterns matching well with the standard fcc lattice structure of silver (JCPDS 04-0783) denoted by the small vertical bars at the bottom in Fig. 2D. Curves (a) and (b) in Fig. 2D represent the diffraction patterns obtained from AgNPs synthesized using laser and UV-A lamp, respectively. The particle size of the AgNPs was also calculated from these XRD patterns. From the peak widths of (111) Bragg diffraction peaks and using Scherrer equation [35], the size of AgNPs synthesized using laser and UV-A lamp was calculated to be ca. $9.5 \mathrm{~nm}$ and ca. $17 \mathrm{~nm}$, respectively. These values are in close agreement with TEM data.

While, with the above two methods it was possible to directly synthesize nanoparticles within a specific size range, it would also be worth, having the possibility to extract nanoparticles of specific size range from a polydisperse sample. This is of particular relevance for AgNPs as most of the common AgNPs synthesis protocols often yield polydisperse sample. Single step size selective extraction of nanoparticles by phase transfer from a polydisperse samples is already well demonstrated [40]. However, extracting more than one size fractions can dramatically improve the cost effectiveness of the process by minimizing the wastage. To achieve this goal we attempted to phase transfer photoreduced AgNPs into different organic solvents using octadecylamine (ODA) as the phase transfer agent. In our preliminary attempts we observed that on changing the solvent for phase transfer, there appeared to be different cut off values with respect to the size of NPs. We employed hexane and chloroform for the phase transfer experiments. AgNPs were synthesized using a similar photoreduction method, except that they were carried out using a $150 \mathrm{~W}$ medium pressure Hg lamp. The synthesis was carried out in non-optimal conditions resulting in polydisperse NPs. Because of the higher intensity of the $150 \mathrm{~W}$ lamp as compared to $4 \mathrm{~W}$ lamp, larger quantities of AgNPs could be readily made within half an hour. The NPs so synthesized were characterized by UV-vis spectroscopy (curve a, Fig. 3). From the TEM measurements (Fig. 4A) it can be seen that the NPs are quite polydisperse, with a considerable population within the $10-15 \mathrm{~nm}$ size range. However, a large number of AgNPs less than $6 \mathrm{~nm}$ can also be noticed. In the TEM particles size distribution (panel a,

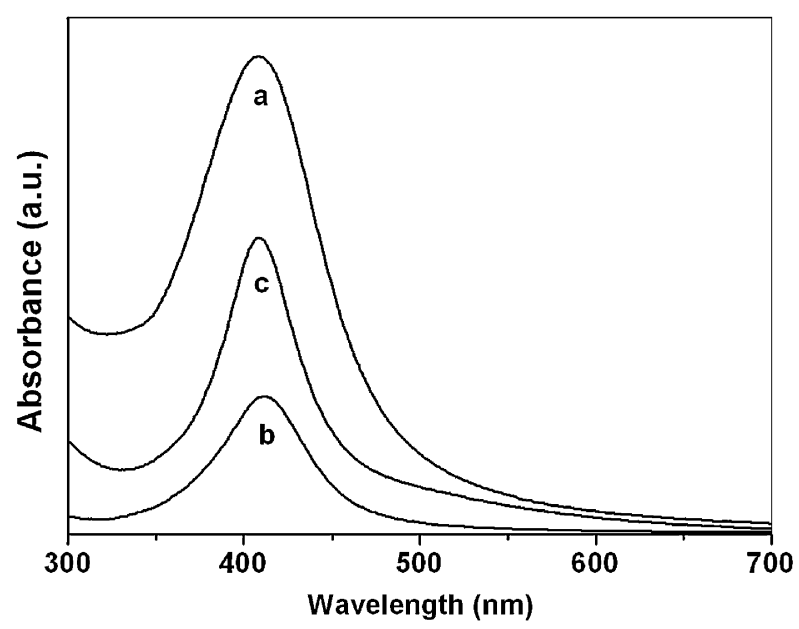

Fig. 3. UV-vis spectra of (curve a) AgNPs synthesized after 30 min of irradiation with a medium pressure Hg lamp, (curve b) phase transferred AgNPs fraction in hexane and (curve c) phase transferred AgNPs fraction in chloroform. 

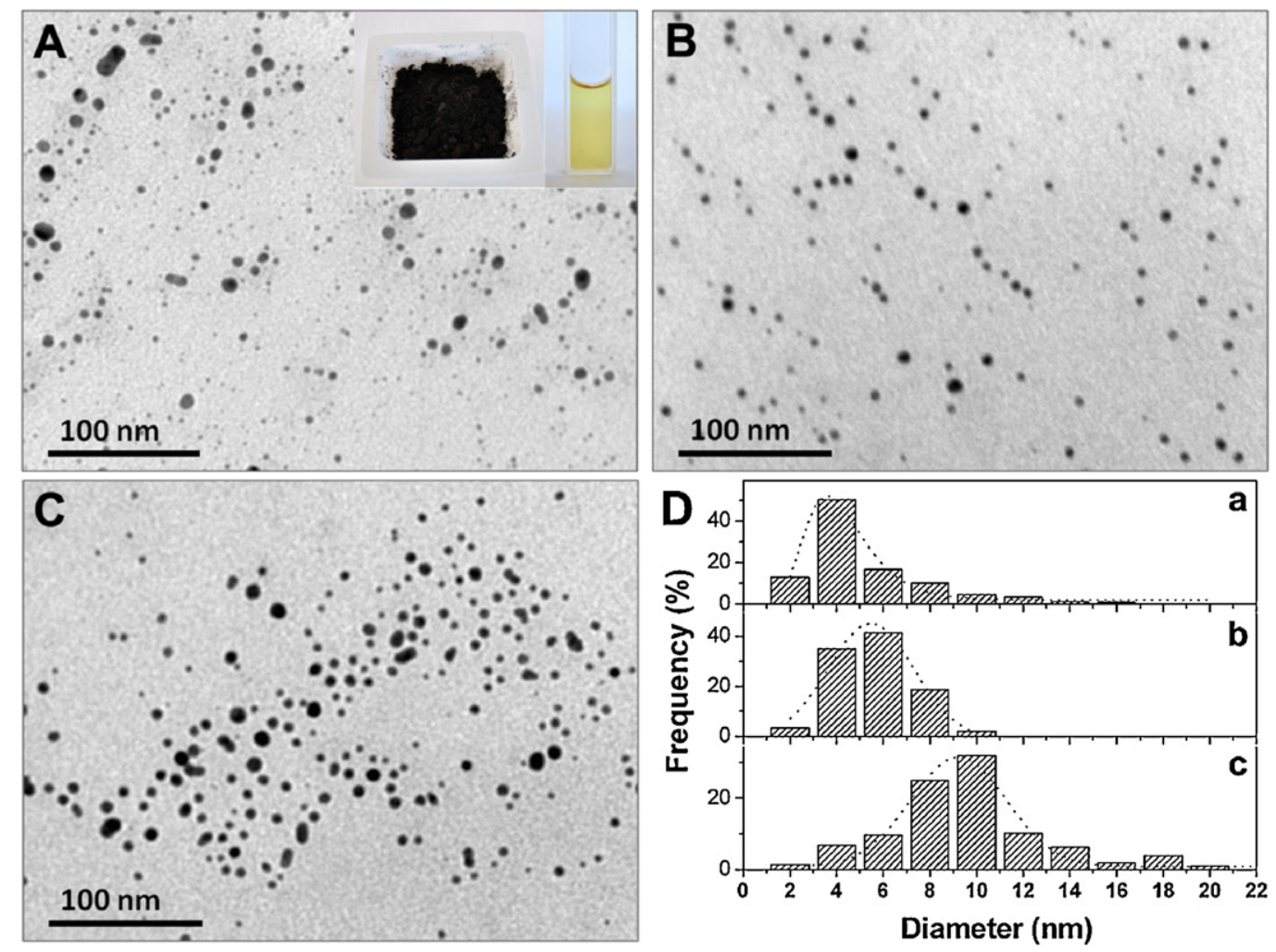

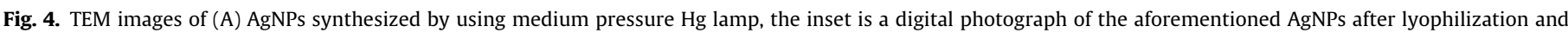

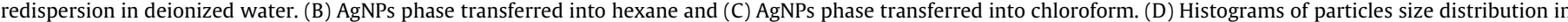
panels a, b and c correspond to the AgNPs in images A, B and C, respectively.

Fig. 4D), it can be observed that, though polydisperse, a large number of very small AgNPs are formed by this method. The histogram is skewed positively rather than a normal distribution and from the size distribution it can be noticed that nearly $79 \%$ of the NPs were of size less than $7 \mathrm{~nm}$. The mean value with standard error from its lognormal fit was $\sim 4 \pm 0.1 \mathrm{~nm}$. As these NPs are quite polydisperse with NPs of broad size range, they are good candidates for testing size fractionation. Apart from these size variations, the AgNPs synthesized by both the UV lamps were observed to be very stable. Because of their high stability these NPs could, in fact, be lyophilized into a dry powder that could be readily redispersed in water without aggregation (inset of Fig. 4A). We believe that this enhanced stability has tremendous significance for the bulk synthesis of silver nanoparticles for commercial production. Currently, silver nanoparticles in powder form in the presented size range are commercially sold with large excess of polymer drastically depleting the percentage of actual silver content in it. Further, it could also be counter productive where a thinner interface between the particle and host matrix is necessary. After synthesis, the $\mathrm{pH}$ of the solution was measured to be 8 and the $\zeta$ potential was $-29 \mathrm{mV}$. As the particles are highly negatively charged, ODA was used for the phase transfer experiments. Under proper pH conditions, the amine functional group of the ODA molecule will become positively charged on protonation and facilitate the extraction of NPs by electrostatic interaction [41].

To a $50 \mathrm{~mL}$ of as prepared aqueous solution of AgNPs synthesized using the $150 \mathrm{~W}$ UV lamp, $50 \mathrm{~mL}$ of $2 \times 10^{-4} \mathrm{M}$ ODA in hexane was added, followed by addition of $200 \mu \mathrm{L}$ of $1 \mathrm{M} \mathrm{HCl}$ and then agitated vigorously for few minutes. A yellow color could be readily observed in the upper organic phase while the lower aqueous phase was still colored implying that only partial phase transfer was achieved. Additionally, a slight brown color film was also present at the interface of the two solutions. The NPs at the interface are 2D aggregates that are not phase transferred into the organic phase due to insufficient hydrophobization $[40,42]$. By increasing the concentration of ODA in hexane or by increasing the amount of added $\mathrm{HCl}$, no further increase in the phase transfer of AgNPs could be effected. On addition of $\mathrm{HCl}$, the aqueous phase was measured to have $\mathrm{pH}$ 3 and the $\zeta$ potential of AgNPs decreased in magnitude to $-15 \mathrm{mV}$. As the pka value for the carboxyl group of tyrosine is 2.33 [43], the nanoparticles surface would continue to be negatively charged due to the deprotonation of the surface bound carboxyl group. We believe that the residual surface charge after addition of $\mathrm{HCl}$ though not enough to stabilize them in the aqueous medium for long, is nevertheless sufficient to facilitate phase transfer. Curve b in Fig. 3A shows the spectrum of the AgNPs phase transferred into the hexane phase. The low absorbance peak, corresponding to the surface plasmon resonance of silver, was broad and observed to be at $411 \mathrm{~nm}$. To visualize the particles that were phase transferred to the hexane phase, TEM imaging was done on these samples (Fig. 4B). From the images, a decreased polydispersity of the AgNPs can be readily noticed as compared to the AgNPs in the parent aqueous solution (Fig. 4A). It can also be noticed that the AgNPs only below the size of $10 \mathrm{~nm}$ have been phase transferred into the hexane phase. In panel b of Fig. 4D the particles size distribution calculated from the TEM images of AgNPs phase transferred to the hexane phase is shown. The calculated mean size was $5.5 \pm 1.8 \mathrm{~nm}$, and the entire NPs were within a comparatively narrower size range, implying a selective phase transfer of smaller size NPs with a significantly improved size dispersion. Similar selective phase transfer of gold NPs below $10 \mathrm{~nm}$ size was also reported by Cheng et al [40]. It may be further observed that the average size of AgNPs seem to be slightly increased which is quite probable due to some aggregation during ligand exchange with the surfactant molecules in a 
phase transfer process [44]. However, such ligand exchange would only be a minor component as the phase transferred AgNPs were observed by FTIR measurements to be extracted along with the capping tyrosine derivatives. Post phase separation of smaller size AgNPs, usually, the residual nanoparticles in the aqueous phase irreversibly assemble at the interface as discussed above and cannot be extracted further by a second phase transfer step. As the irreversible aggregation at the interface is due to the charge neutralization [40,42], and given that the AgNPs reported here are quite stable, we expected to suppress extensive irreversible aggregation and facilitate a second extraction. We therefore, attempted to carry out a second cycle of phase transfer by using ODA in chloroform $\left(50 \mathrm{~mL}, 1 \times 10^{-4} \mathrm{M}\right)$ as the new organic medium. On vigorous agitation of these two phases, it was indeed possible to phase transfer the remaining AgNPs from the aqueous medium. A clear yellow color was observed in the $\mathrm{CHCl}_{3}$ phase and now the aqueous phase was completely colorless, unlike in the case of phase transfer with ODA-hexane. Additionally, at the interface between the two phases, the brown color of the film became very faint, indicating that the AgNPs assembled here were also extracted to the $\mathrm{CHCl}_{3}$ phase. Curve c in Fig. $3 \mathrm{C}$ represents the UV-vis spectrum of AgNPs in $\mathrm{CHCl}_{3}$ phase. The absorbance peak was measured to be at $408 \mathrm{~nm}$, close to the peak corresponding to the as prepared aqueous AgNPs solution. However, the peak width is apparently narrower than that observed for the aqueous AgNPs solution. We would like to mention here that the UV-vis spectra for a given metal NPs sample in hexane or chloroform are slightly red-shifted as compared to their spectra in aqueous media due to the change in the refractive index of the medium [45]. Therefore, the observed UV-vis spectra of AgNPs in the $\mathrm{CHCl}_{3}$ phase should correspond to a relatively blue shifted absorption band with respect to $408 \mathrm{~nm}$ in aqueous medium. From the TEM image of the AgNPs in $\mathrm{CHCl}_{3}$ phase (Fig. 4C), a large number of AgNPs around $10 \mathrm{~nm}$ size can be observed and indeed have a narrow size distribution with respect to the parent aqueous solution. Further, AgNPs of size less than $6 \mathrm{~nm}$ are sparsely present, signifying that the very small NPs were well extracted into hexane phase. In panel $\mathrm{c}$ of Fig. $4 \mathrm{D}$, the particles size distribution in $\mathrm{CHCl}_{3}$ phase is shown. In absence of the small NPs, the NPs of size around $10 \mathrm{~nm}$ that were only a minor component in the as prepared AgNPs solution, now begin to appear with a higher frequency. It may however be observed that the AgNPs in $\mathrm{CHCl}_{3}$ phase seem to have some particles bigger than that observed in the parent solution and could be due to some unavoidable aggregation of the AgNPs, especially of those at the liquid-liquid interface. The mean particles size from the fitted Gaussian curve was calculated to be $9.2 \mathrm{~nm} \pm 2 \mathrm{~nm}$. The AgNPs phase transferred to both hexane and $\mathrm{CHCl}_{3}$ phases could easily be dried in rotary evaporator and redispersed in either solvent. It has thus been demonstrated that a NPs sample with broad polydispersion can be well separated into two different narrow size ranges. The ability of chloroform to facilitate phase transfer of larger size NPs as compared to hexane can be attributed to its relatively higher polarity [42], It has been well observed that the ion pair association increases with increase in the solvent dielectric constant and have also been observed to significantly improve the extraction of ionic species from aqueous medium [46]. We believe that a similar improved ion pairing occurs between protonated octadecylamine molecule and the negatively charged nanoparticles at the liquid-liquid interface when changing the solvent from hexane to chloroform. This improved ion pairing could possibly facilitate in shifting the size limit that can be extracted into the organic phase. Therefore, with hexane, nanoparticles up to a size of $6 \mathrm{~nm}$ could be conveniently extracted, with chloroform, nanoparticles of size around $10 \mathrm{~nm}$ could also be extracted. Here, we have therefore used solvent with different polarity, while keeping the same phase transfer agent to selectively extract NPs within a particular size range from the aqueous phase.

\section{Conclusions}

We have demonstrated a photoreduction method to synthesize AgNPs in aqueous medium with a narrow size distribution using tyrosine as a photoreducing agent and an effective stabilizing agent. By changing the source of the light, the size of the AgNPs could be well controlled. We have also demonstrated size fractionation of a polydisperse sample of aqueous AgNPs into two different size range by applying phase transfer method. The additional factor that facilitates such sequential fractionation of the size selected NPs is the high stability of the tyrosine stabilized AgNPs even in absence of any thiols or polymers.

\section{Acknowledgment}

We thank Paolo Cazzato for the technical assistance to conduct the laser experiments.

\section{Appendix A. Supplementary data}

Supplementary data associated with this article can be found, in the online version, at doi:10.1016/j.colsurfa.2011.10.003

\section{References}

[1] M. Pelton, J. Aizpurua, G. Bryant, Laser Photonics Rev. 2 (2008) 136-159.

[2] C.N.R. Rao, G.U. Kulkarni, P.J. Thomas, P.P. Edwards, Chem. Eur. J. 8 (2002) 28-35.

[3] O.S. Ivanova, F.P. Zamborini, J. Am. Chem. Soc. 132 (2009) 70-72.

[4] C.C. Yang, S. Li, Phys. Rev. B 75 (2007) 165413.

[5] W. Luo, L. Deng, K. Su, K. Li, G. Liao, S. Xiao, Physica B 406 (2011) 859-863.

[6] P.K. Jain, X. Huang, I.H. El-Sayed, M.A. El-Sayed, Acc. Chem. Res. 41 (2008) 1578-1586.

[7] A.N. Shipway, E. Katz, I. Willner, ChemPhysChem 1 (2000) 18-52.

[8] O. Yehezkeli, R. Tel-Vered, S. Raichlin, I. Willner, ACS Nano 5 (2011) 2385-2391.

[9] J.A. Rodriguez, Catal. Today 160 (2011) 3-10.

[10] S.S. Shankar, S. Deka, Sci. Adv. Mater. 3 (2011) 169-195.

[11] S. Preciado-Flores, D.A. Wheeler, T.M. Tran, Z. Tanaka, C. Jiang, M. BarbozaFlores, F. Qian, Y. Li, B. Chen, J.Z. Zhang, Chem. Commun. 47 (2011) 4129-4131.

[12] Y. Lin, M. Yin, F. Pu, J. Ren, X. Qu, Small 7 (2011) 1557-1561.

[13] A.N. Kyung-Min Lee, Neogi Purnima Basu, Kim Minjung, Kim Bongsoo, Luchowski Rafal, Gryczynski Zygmunt, Calander Nils, Choi Tae-Youl, J. Biomed. Opt. 16 (2011) 056008

[14] G. Rong, H. Wang, B.r.M. Reinhard, Nano Lett. 10 (2009) 230-238.

[15] A. Kumar, P.K. Vemula, P.M. Ajayan, G. John, Nat. Mater. 7 (2008) 236-241.

[16] T. Mitsudome, A. Noujima, Y. Mikami, T. Mizugaki, K. Jitsukawa, K. Kaneda, Angew. Chem. Int. Ed. 49 (2010) 5545-5548.

[17] A. Royon, K. Bourhis, M. Bellec, G. Papon, B. Bousquet, Y. Deshayes, T. Cardinal, L. Canioni, Adv. Mater. 22 (2010) 5282-5286.

[18] V.N. Richards, N.P. Rath, W.E. Buhro, Chem. Mater. 22 (2010) 3556-3567.

[19] P. Jeevanandam, C.K. Srikanth, S. Dixit, Mater. Chem. Phys. 122 (2010) $402-407$.

[20] X.Z. Lin, A.D. Terepka, H. Yang, Nano Lett. 4 (2004) 2227-2232.

[21] D.S. Sidhaye, B.L.V. Prasad, New J. Chem. 35 (2011) 755-763.

[22] R. Shankar, B.B. Wu, T.P. Bigioni, J. Phys. Chem. C 114 (2010) 15916-15923.

[23] S. He, J. Yao, P. Jiang, D. Shi, H. Zhang, S. Xie, S. Pang, H. Gao, Langmuir 17 (2001) 1571-1575.

[24] Q. Zhang, J. Xie, Y. Yu, J.Y. Lee, Nanoscale 2 (2010) 1962-1975.

[25] M. Iida, C. Baba, M. Inoue, H. Yoshida, E. Taguchi, H. Furusho, Chem. Eur. J. 14 (2008) 5047-5056.

[26] B. Hu, S.-B. Wang, K. Wang, M. Zhang, S.-H. Yu, J. Phys. Chem. C 112 (2008) 11169-11174.

[27] J.-P. Malval, M. Jin, L. Balan, R.l. Schneider, D.-L. Versace, H.l.n. Chaumeil, A. Defoin, O. Soppera, J. Phys. Chem. C 114 (2010) 10396-10402.

[28] G.-n. Xu, X.-l. Qiao, X.-l. Qiu, J.-g. Chen, Colloid Surf. A 320 (2008) 222-226.

[29] F. Mafuné, J.-y. Kohno, Y. Takeda, T. Kondow, H. Sawabe, J. Phys. Chem. B 104 (2000) 8333-8337.

[30] L. Liu, T. Wei, X. Guan, X. Zi, H. He, H. Dai, J. Phys. Chem. C113(2009) 8595-8600.

[31] B. Chen, X. Jiao, D. Chen, Cryst. Growth Des. 10 (2010) 3378-3386.

[32] D.D. Evanoff, G. Chumanov, J. Phys. Chem. B 108 (2004) 13948-13956.

[33] P.R. Selvakannan, A. Swami, D. Srisathiyanarayanan, P.S. Shirude, R. Pasricha, A.B. Mandale, M. Sastry, Langmuir 20 (2004) 7825-7836.

[34] S. Si, R.R. Bhattacharjee, A. Banerjee, T.K. Mandal, Chem. Eur. J. 12 (2006) $1256-1265$.

[35] H.P. Klug, L.E. Alexander, X-ray Diffraction Procedures: For Polycrystalline and Amorphous Materials, 2nd ed., John Wiley \& Sons, New York, 1974.

[36] W.C. Bell, M.L. Myrick, J. Colloid Interface Sci. 242 (2001) 300-305.

[37] J. Feitelson, E. Hayon, A. Treinin, J. Am. Chem. Soc. 95 (1973) 1025-1029. 
[38] D.V. Bent, E. Hayon, J. Am. Chem. Soc. 97 (1975) 2599-2606.

[39] J. Jortner, M. Ottolenghi, G. Stein, J. Am. Chem. Soc. 85 (1963) 2712-2715.

[40] W. Cheng, E. Wang, J. Phys. Chem. B 108 (2004) 24-26.

[41] M. Sastry, M. Rao, K.N. Ganesh, Acc. Chem. Res. 35 (2002) 847-855.

[42] M. Karg, N. Schelero, C. Oppel, M. Gradzielski, T. Hellweg, R. von|Klitzing, Chem. Eur. J. 17 (2011) 4648-4654.
[43] Y. Henchoz, J. Schappler, L. Geiser, J. Prat, P.-A. Carrupt, J.-L. Veuthey, Anal Bioanal. Chem. 389 (2007) 1869-1878.

[44] N. Lala, S.P. Lalbegi, S.D. Adyanthaya, M. Sastry, Langmuir 17 (2001)3766-3768

[45] S. Underwood, P. Mulvaney, Langmuir 10 (1994) 3427-3430.

[46] D. Quintanar-Guerrero, E. Allémann, H. Fessi, E. Doelker, Pharm. Res. 14 (1997) 119-127. 